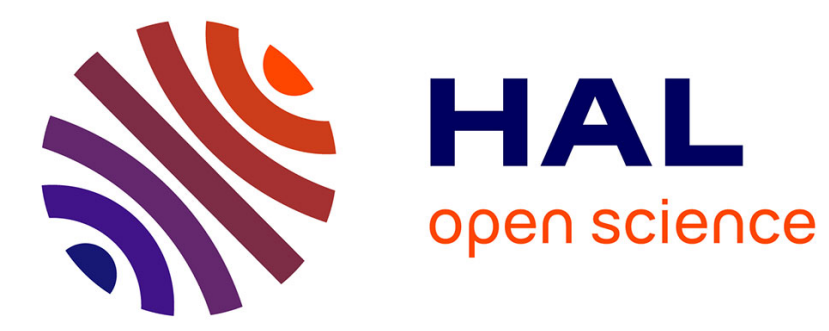

\title{
La trajectoire, une autre approche des effets de la rénovation
}

\author{
Christine Lelévrier
}

\section{To cite this version:}

Christine Lelévrier. La trajectoire, une autre approche des effets de la rénovation. Fol Sylvie; Miot Yohan; Vignal Cécile. Mobilités résidentielles, territoires et politiques publiques, Presses Universitaires du Septentrion, pp.119-138, 2014. hal-01198709

\section{HAL Id: hal-01198709 \\ https://hal.science/hal-01198709}

Submitted on 2 Mar 2016

HAL is a multi-disciplinary open access archive for the deposit and dissemination of scientific research documents, whether they are published or not. The documents may come from teaching and research institutions in France or abroad, or from public or private research centers.
L'archive ouverte pluridisciplinaire HAL, est destinée au dépôt et à la diffusion de documents scientifiques de niveau recherche, publiés ou non, émanant des établissements d'enseignement et de recherche français ou étrangers, des laboratoires publics ou privés. 
Lelévrier C., 2014, «La trajectoire, une autre approche des effets de la rénovation », Fol S., Miot Y., Vignal C. (dir.), Mobilités résidentielles, territoires et politiques publiques, Lille, Presses Universitaires du Septentrion, 119-138.

\title{
La trajectoire, une autre approche des effets de la rénovation
}

\author{
Christine Lelévrier \\ IUP, Université Paris-Est, Lab’Urba
}

En 1970, deux sociologues français analysaient les rapports de cohabitation dans un «grand ensemble » de la région parisienne, à l'aune des trajectoires résidentielles des groupes sociaux en présence (Chamboredon, Lemaire, 1970). Cette approche dynamique affinait l'analyse des différenciations internes aux classes sociales et montrait comment des filières d'attribution rassemblaient de manière contrainte des populations hétérogènes, dont certaines n'étaient que de passage tandis que pour d'autres, l'arrivée dans ce grand ensemble marquait l'aboutissement de leur trajectoire. Mais elle faisait surtout de la diversité des trajectoires, et en particulier des possibilités de départ, un facteur déterminant des "attitudes à l'égard du grand ensemble " et des rapports de voisinage. Au fur et à mesure de la paupérisation de ces grands ensembles et en dehors de quelques travaux menés dans les années 1990 (Peraldi, Foret, 1992; Vieillard-Baron, 1998), l'analyse des trajectoires propres à ce type d'ensemble résidentiel est devenue de plus en plus marginale. Avec le départ des classes moyennes et la montée de la précarité de l'emploi paupérisant ces grands ensembles, les recherches se sont plutôt déplacées vers la concentration de pauvreté, révélatrice des transformations de la classe ouvrière (Beaud, Pialoux, 2003) ou productrice d'effets de quartier négatifs sur les trajectoires sociales des populations et la réputation des lieux (Goux et Maurin, 2007 ; Sari, 2012).

Trente ans après leur construction, les programmes de démolition-reconstruction de ces "grands ensembles" reposent à leur manière la question du peuplement et des trajectoires résidentielles et redonnent tout son intérêt à ce type d'approche. Tout d'abord, les politiques publiques de régénération ou de rénovation, mises en place en France mais également dans d'autres pays pour réduire la concentration de pauvreté, ont des effets directs et importants sur la mobilité des ménages. Ces politiques prévoient en effet de démolir des tours et des barres de logements sociaux pour les remplacer par de petites résidences, pour partie privées, censées favoriser un peuplement plus mixte, devant lui-même entraîner une sorte de cercle vertueux, lié notamment à l'arrivée de classes moyennes (Bolt et al., 2010 ; Donzelot, 2012 ; Kleinhans, 2004). Ensuite, l'action publique se donne pour objectif d'améliorer "la qualité des parcours résidentiels", proposant même dans des chartes locales de faire du relogement du locataire « un moment privilégié dans son parcours résidentiel pour l'aider à préciser ses choix et vivre ce relogement comme une "seconde chance" ${ }^{2}$. Enfin, ces transformations du cadre résidentiel recomposent aussi les conditions de cohabitation et les configurations de voisinage. Au regard de ces logiques de l'action, les habitants de ces quartiers pourraient voir leur trajectoire résidentielle et sociale changer au moins de trois manières: en déménageant sur place mais en bénéficiant ou pas des « opportunités résidentielles » de la rénovation (nouveaux logements, relocalisations...); en étant relogés ailleurs mais dans des environnements sociaux plus ou moins mixtes; en bénéficiant au travers d'interactions sociales, de la proximité spatiale de nouvelles classes moyennes, porteuses de normes et de valeurs censées être plus intégratrices ou de l'amélioration du cadre de vie. Pourtant, la question de savoir si ces changements vont bénéficier aux populations locales, et en particulier aux plus pauvres d'entre eux, reste posée par les chercheurs (Tunstall, Fenton, 2006).

À l'heure où les bilans du Programme National de Rénovation Urbaine mis en place en 2003 en France mettent surtout l'accent sur la difficulté, voire l'impossibilité de réaliser la mixité sociale

1 Extrait du règlement général de l'ANRU, 2007.

2 Extrait de la charte de relogement d'un site de Seine-et-Marne in Lelévrier C., Noyé C., 2007. 
(CES ANRU, 2011; Cour des comptes, 2012), l'analyse en termes de trajectoires, assez peu développée dans la recherche, permet de décaler le regard sur les effets de l'action, et de manière plus générale sur ces quartiers et les rapports que ces «mobiles », ceux qui arrivent, ceux qui partent et ceux qui sont déplacés, entretiennent avec leur lieu de résidence. Quels bénéfices, avantages ou désavantages les destinataires de ces politiques vont-ils en retirer? Comment analyser les effets de cette intervention publique sur les trajectoires sociales et résidentielles des populations concernées, qu'elles soient contraintes par les démolitions ou plus choisies dans le cadre des nouveaux logements privés, et pas seulement sur la diversité de l'habitat ou sur la composition sociale des quartiers rénovés?

Cet article se propose d'apporter quelques éclairages sur ces questions en analysant les mobilités des populations relogées et leur vécu des changements résidentiels. Cette approche compréhensive replace le relogement dans la temporalité longue des étapes successives de la trajectoire (Authier, 2010; Grafmeyer, 1994) tout en reprenant l'hypothèse que la diversité des trajectoires résidentielles détermine les attitudes et perceptions différentes de ce relogement. Nous nous appuierons principalement sur les résultats de recherches empiriques menées dans sept quartiers franciliens et plus particulièrement sur cent vingt entretiens qualitatifs menés auprès de ménages relogés. Nous reviendrons d'abord sur l'approche en termes de trajectoires tout en précisant quelques éléments méthodologiques. Nous exposerons ensuite les résultats de recherche en deux temps : la présentation des trois types de trajectoires résidentielles identifiées dans ce travail et la manière dont elles peuvent constituer un cadre d'analyse pour comprendre le sens de ce changement pour les ménages et les effets de l'action.

\section{Trajectoires et action publique}

Le choix du terme de trajectoire n'est pas neutre. Il permet d'abord de ne pas reprendre la catégorie pratique du parcours résidentiel, utilisée par les acteurs de l'habitat et développée dans le programme de rénovation urbaine. Ce parcours résidentiel désigne alors deux déplacements entre un logement et un autre, un quartier et un autre. La notion de trajectoire correspond à l'ensemble des "séquences résidentielles qui s'enchaînent au cours de la vie d'un ménage (Grafmeyer, 1994). D'une part, elle s'inscrit dans la temporalité longue de l'histoire des individus, établissant des formes de causalité entre les étapes résidentielles et d'autres évènements familiaux et professionnels. D'autre part, elle sous-entend davantage que d'autres termes, l'idée d'une direction, d'un " ordre intelligible », faisant ainsi "l'hypothèse que les mobilités ont un sens" (Grafmeyer, op. cit., p. 67). La notion de trajectoire renvoie à cette triple dimension de processus, de temporalité et de sens et permet de croiser les «déterminants», les «stratégies personnelles》 et les «évènements inattendus» qui peuvent l'influencer (Charbonneau, 1998). Comment analyser les liens entre trajectoire et action publique ?

On peut aborder la question des effets des mobilités sur les trajectoires résidentielles des populations en objectivant des changements dans les caractéristiques de taille et de confort du logement, dans la dépense consacrée à ce logement ou encore dans la composition sociale des espaces résidentiels occupés (le «type résidentiel» défini par Lévy, 1998). Ces changements peuvent permettre de qualifier les trajectoires d'«ascendantes» ou de «promotionnelles», de «descendantes» et de "neutres », selon que la situation s'améliore, se dégrade ou reste inchangée (Lévy, op. cit., 1998). La mobilité contrainte liée à l'événement inattendu et non anticipé de la démolition reste toutefois difficilement comparable à une mobilité ordinaire pour au moins trois raisons. Tout d'abord, elle ne relève pas directement de changements professionnels et sociaux et des étapes d'un cycle de vie devenu lui-même de plus en plus chaotique avec les transformations des modèles familiaux et du contexte économique (Bonvalet, Fribourg, 1990). Ensuite, la notion de choix résidentiel a du sens pour les nouveaux arrivants mais reste assez inappropriée pour les ménages relogés, même s'ils peuvent avoir une marge de manœuvre (Lelévrier, Noyé, 2012). Enfin, une des différences importantes tient à l'encadrement de ces mobilités par les bailleurs sociaux et les villes, qui constitue un cadre d'interaction sociale et de normalisation des situations résidentielles.

Dans le même temps, cet événement de la démolition et l'émergence d'une nouvelle offre d'habitat dans et autour des quartiers rénovés ouvrent des perspectives nouvelles pour les ménages. Ces derniers vont bien sûr les saisir différemment selon leur propre capacité et aspiration à la mobilité mais également selon le moment où se situe cet «événement» dans leur trajectoire. Ainsi, on peut 
supposer que la mobilité contrainte du relogement peut interagir avec d'autres projets résidentiels et les accélérer en offrant des opportunités de les concrétiser, voire en déclencher au vu des avantages offerts (incitation financière de l'État au relogement de la moitié des ménages dans du neuf, construction de programmes d'accession à taxes réduites, logement plus grand...). À l'inverse, on peut également considérer que la démolition peut venir déstabiliser des situations, soit du côté des conditions de logement (taille, loyer) soit du côté de réseaux familiaux et sociaux de voisinage et de quartier qui constituent autant de points d'ancrage et de ressources pour les plus pauvres (Fol, 2009). Même s'il n'y a pas de choix résidentiel, le fait que les ménages doivent réagir à trois propositions en moyenne de logements les met en situation d'arbitrage (Vignal, 2005) et souvent de réflexivité sur leur logement et leur quartier. Qu'en est-il de l'approche plus subjective des ménages sur le sens de ce relogement et les effets sur leurs réseaux ?

Les traces de l'histoire plus ancienne de la rénovation des années 1960 sont encore présentes, laissant planer le spectre d'un "délogement» détruisant les réseaux sociaux des classes populaires tandis que l'arrivée de classes moyennes extérieures participaient d'une « gentrification » des quartiers ouvriers (Coing, 1966 ; Dansereau, 1985). Mais ces approches avaient peu développé de véritables suivis de cohortes permettant d'affirmer pleinement ces effets négatifs. Les travaux d'Henri Coing restaient d'ailleurs assez nuancés dès qu'il s'agissait d'apprécier les changements au regard des situations individuelles. Les résultats disponibles sur la rénovation engagée depuis une quinzaine d'années en France et dans d'autres pays montrent à l'évidence que le contexte n'est pas le même et ils restent assez mitigés. Certes, le déplacement serait toujours aussi porteur de traumatisme et de stress pour des populations qui n'ont pas choisi de bouger et qui se retrouveraient plutôt dans des quartiers aussi pauvres, notamment aux États-Unis (Allen, 2000 ; Goetz, 2002 ; Varady, Walker, 2000) tout en perdant leurs réseaux communautaires (Vankatesh, Celimli, 2004). En France, les mobilités conduiraient plutôt à re-concentrer les populations les plus pauvres dans les mêmes quartiers et les mêmes immeubles (Faure, 2006 ; Lelévrier, 2010). La synthèse de plusieurs recherches (Lelévrier, 2008) montrait des résultats convergents dans les relogements. Les mobilités de proximité maintenaient $80 \%$ des ménages dans la même commune et $50 \%$ dans le même quartier. De plus, les ménages relogés dans des quartiers plus aisés comme à Lyon y restaient cantonnés dans des secteurs de logements sociaux dont le peuplement n'était pas très différent (Harzo, Lauriac, Foret, Bidet, 2007).

Aux Pays-Bas comme en France, certains travaux nuancent les effets négatifs du relogement en mettant en avant d'une part la satisfaction des locataires (Kleinhans, Van der Laan Bouma-Doff, 2008 ; Oblet, Villechaise, 2009), d'autre part, la notion «d'opportunités résidentielles » saisies à la fois par les relogés et par les habitants qui accèdent à la propriété dans leur quartier (Kleinhans, 2003 ; Lelévrier, Noyé, 2012; Bolt et al., op.cit.). Mais à l'évidence, les "grandes familles", les « étrangers » et, dans certains sites, les personnes très âgées, ont des trajectoires plus subies que choisies (Huynh, 2008). En dehors de quelques travaux (Faure, 2006 ; Gilbert, 2012), la plupart de ces approches s'intéressent avant tout à l'étape du relogement (avant/après), autrement dit à la trajectoire comme un effet direct de l'action. Notre approche compréhensive des trajectoires résidentielles longues, incluant l'ensemble des séquences résidentielles depuis le premier logement autonome, permet de saisir la diversité des expériences et du vécu des changements. Autrement dit, la trajectoire n'est pas ou pas seulement un effet de l'action mais un instrument d'analyse du vécu de ces changements par les populations.

\section{Le relogement, une étape dans trois types de trajectoires résidentielles}

Nous avons mené plusieurs recherches successives dans sept sites franciliens avec les mêmes méthodes entre 2004 et 2006 : une analyse statistique des mobilités à partir de fichiers de bailleurs et des entretiens qualitatifs menés auprès d'un échantillon de ménages choisis parmi l'ensemble des ménages relogés, de manière à avoir une diversité de mobilités et de types de ménages (âge, composition familiale, revenus). Notre démarche de construction typologique est restée empirique, pouvant s'apparenter à une classification plutôt inductive. Les trois types de trajectoires mis en évidence sont construits autour de trois variables : le moment où se situe le relogement (en début, au milieu ou en fin de trajectoire) ; la situation socio-économique des ménages (ressources et composition familiale) et les circonstances de l'arrivée et la durée de résidence dans le quartier avant le relogement. Stabilité, vulnérabilité ou incertitude des destins sociaux et professionnels caractérisent les trois types 
de trajectoires socio-professionnelles et résidentielles. Nous avons analysé dans ces trajectoires le rapport au quartier, qui module d'autant l'envie des ménages de partir ou de rester (Authier, op. cit.) et fait varier également les ressources mobilisables pour « être relogé » ou " se reloger », les perceptions et les expériences du relogement.

Par processus de relogement, nous entendons l'ensemble des étapes d'un déménagement contraint par la démolition ou la transformation d'un immeuble, mais sous contrôle de l'action publique. Ce relogement est encadré par les agents et structures chargés du relogement, internes au bailleur ou aux villes et associant le plus souvent les gestionnaires des bâtiments démolis et les services communaux au sein d'équipes de Maîtrise d'œuvre Urbaine et Sociale (MOUS). Ces étapes vont de l'annonce de la démolition à l'installation dans le nouveau logement du ménage déplacé, en passant par toute une série d'interactions amenant le ménage à des arbitrages et négociations en fonction de ses propres contraintes économiques et familiales, de celles de l'offre et de la gestion locales (propositions, vacance, rotation, logements neufs reconstruits....).

Tableau 19 : Sites et nombre d'entretiens menés

\begin{tabular}{|l|l|l|l|}
\hline Opérations & $\begin{array}{l}\text { Nombre de logements } \\
\text { démolis }\end{array}$ & $\begin{array}{l}\text { Nombre de ménages dans les } \\
\text { fichiers bailleurs } \\
\text { (base statistique) }\end{array}$ & $\begin{array}{l}\text { Nombre d'entretiens } \\
\text { réalisés }\end{array}$ \\
\hline Site 1 & 960 & 627 & 35 \\
\hline Site 2 & 1121 & 730 & 26 \\
\hline Site 3 & 344 & 255 & 15 \\
\hline Site 4 & 196 & 92 & 9 \\
\hline Site 5 & 166 & 52 & 13 \\
\hline Site 6 & 135 & 91 & 13 \\
\hline Site 7 & 273 & 238 & 10 \\
\hline Ensemble & 3195 & 2085 & 121 \\
\hline
\end{tabular}

Tableau 20 : Caractéristiques des ménages par types de trajectoires

\begin{tabular}{|c|c|c|c|c|c|}
\hline Type & $\begin{array}{l}\text { Nombre } \\
\text { de } \\
\text { ménages }\end{array}$ & $\begin{array}{l}\text { Age du } \\
\text { chef de } \\
\text { ménage }\end{array}$ & $\begin{array}{l}\text { Situation } \\
\text { emploi et } \\
\text { ressources }\end{array}$ & $\begin{array}{l}\text { Type et taille } \\
\text { du ménage }\end{array}$ & trajectoire \\
\hline $\begin{array}{l}\text { Type } 1 \\
\text { Trajectoires } \\
\text { stabilisées de } \\
\text { « vieilles } \\
\text { familles } \\
\text { ouvrières » }\end{array}$ & 31 & $\begin{array}{l}\text { Plus de } \\
50 \text { ans }\end{array}$ & $\begin{array}{l}\text { Retraités, } \\
\text { emplois } \\
\text { stables } \\
\text { (ouvrier, } \\
\text { artisan) }\end{array}$ & $\begin{array}{l}\text { Petits ménages } \\
\text { (moins de } 5 \\
\text { personnes) } \\
\text { Couple avec } \\
\text { grands enfants, } \\
\text { couples sans } \\
\text { enfant, isolés }\end{array}$ & $\begin{array}{l}\text { Migration de province ou de } \\
\text { l'étranger dans les années } \\
1950 / 1960 \\
\text { Logement social=promotion } \\
\text { Emploi et résidence } \\
\text { (foyer/logement } \\
\text { précaire/logement } \\
\text { social/mutations. Ancrage local }\end{array}$ \\
\hline $\begin{array}{l}\text { Type } 2 \\
\text { Trajectoires } \\
\text { subies de } \\
\text { ménages } \\
\text { « fragilisés et } \\
\text { de } \\
\text { "grandes } \\
\text { familles » }\end{array}$ & 46 & $\begin{array}{l}\text { Tous } \\
\text { âges }\end{array}$ & $\begin{array}{l}\text { Ressources } \\
\text { faibles, } \\
\text { RMI et } \\
\text { prestations, } \\
\text { dettes }\end{array}$ & $\begin{array}{l}\text { Couples avec } \\
\text { plus de trois } \\
\text { enfants, } \\
\text { familles } \\
\text { monoparentales } \\
\text { avec plus de } 2 \\
\text { enfants, isolés }\end{array}$ & $\begin{array}{l}\text { Trajectoires subies de } \\
\text { sédentaires - un logement social } \\
\text { «protecteur » } \\
\text { Mais avec ruptures familiales, } \\
\text { succession de logements privés } \\
\text { et isolement des familles en } \\
\text { précarité } \\
\text { Hébergement et ancrage } \\
\text { communautaire des grandes } \\
\text { familles immigrées }\end{array}$ \\
\hline $\begin{array}{l}\text { Type } 3 \\
\text { Trajectoires } \\
\text { ouvertes, } \\
\text { petits } \\
\text { ménages } \\
\text { actifs à } \\
\text { revenus }\end{array}$ & 44 & $\begin{array}{l}\text { Moins de } \\
40 \text { ans }\end{array}$ & $\begin{array}{l}\text { Un actif au } \\
\text { moins, } \\
\text { emplois } \\
\text { stables } \\
\text { (public), } \\
\text { promotions }\end{array}$ & $\begin{array}{l}\text { Petits ménages } \\
\text { (moins de } 6 \\
\text { personnes) }\end{array}$ & $\begin{array}{l}\text { Début ou milieu de trajectoire, } \\
\text { projets résidentiels, avec des } \\
\text { « enfants de la cité », de jeunes } \\
\text { immigrés et des salariés sans } \\
\text { ancrage local }\end{array}$ \\
\hline
\end{tabular}


\begin{tabular}{|l|l|l|l|l|l|}
\hline modestes & & & & \\
\hline
\end{tabular}

Source : exploitation des 121 entretiens menés dans sept Zones Urbaines Sensibles franciliennes entre 2004 et 2006.

\section{Les trajectoires stabilisées des "vieilles familles ouvrières"}

La première trajectoire associe étroitement une génération, des trajectoires professionnelles et familiales et des trajectoires résidentielles. Les trajectoires stabilisées de "vieilles familles ouvrières », sont celles de ménages âgés de plus de 50 ans, qui sont dans les lieux depuis plus de 20 ans (type $1 ; 25,6 \%$ des ménages interrogés). Les qualificatifs de "stabilisées» et de "vieilles familles » renvoient d'une part à leur âge et leur ancienneté résidentielle dans le quartier, et d'autre part, à une stabilité à la fois professionnelle, familiale, financière et résidentielle. Ces trajectoires retracent le passé d'ouvriers qui ont commencé à travailler dans des emplois peu qualifiés de l'industrie locale dans les années 1950/1960, ont pu bénéficier de la période d'expansion économique et connaître une relative ascension professionnelle. Ces vieilles familles ouvrières ont été des « grandes familles» de plus de trois enfants, et sont aujourd'hui des couples, dont les enfants sont partis du domicile familial, ou des femmes isolées dont le conjoint est décédé. Ces vieilles familles partagent des histoires migratoires mais qui pour une partie d'entre elles, les ont amenées jeunes, d'Algérie, du Maroc ou du Sénégal en France, le plus souvent avec des contrats de travail, et pour d'autres du Nord et du Centre de la France vers la région parisienne, pour trouver ou/et retrouver un emploi.

L'accès au logement social dans le quartier s'est situé entre 1960 et 1970, au début de leur trajectoire, tandis que le relogement intervient à la fin. Ce qu'ils ont vécu avant structure leurs représentations et leurs attentes du relogement. L'arrivée dans le quartier a fait passer ces ménages de logements précaires au statut plus permanent de locataire d'un logement social, plus confortable et plus grand. Les immigrés se sont logés dans le quartier peu de temps après leur arrivée en France, après un passage en foyer ou dans du locatif privé très précaire, trouvé par l'employeur. Ceux qui venaient d'autres régions de France chercher du travail en Ile-de-France, ainsi que les natifs franciliens, sont d'abord passés par du logement précaire de type caravanes, puis logement d'urgence, avant d'obtenir un logement social dans le quartier. Ce premier accès à un logement social «normal », moderne et grand, a été une opportunité et un "plus ». Le quartier a ensuite constitué une ressource résidentielle car ils ont pu facilement changer de logement au fur et à mesure de l'évolution de la taille de leur famille en faisant des demandes auprès du bailleur qu'ils connaissaient bien, renforçant par làmême leur ancrage local.

La période des premiers temps est reconstruite dans leurs propos comme une période idéale et à jamais perdue. "L'avant» des premières années de leur vie dans le quartier devient une sorte de «paradis perdu » (Giraud, 2000), fait d'un partage de normes d'usage de l'espace et d'une sociabilité de liens forts, un quartier communautaire, espace d'une culture ouvrière, familiale et locale (Verret, 1979). Mais ils évoquent tous aussi la même suite de leur histoire collective, moins ascendante, s'apparentant à une sorte de mise en récit de la "dégradation» de leur quartier. Ce récit de la dégradation a un début, qu'ils font tous partir des «années 1990 ». Dans les trajectoires de ces « vieilles familles ouvrières », « le quartier-village » a été déstructuré bien avant la démolition, même si certains ont réussi à maintenir un micro-voisinage de "pairs" à l'échelle d'une cage d'escalier. Pour ces ménages, la nostalgie du passé est une façon de prendre leur distance par rapport à leur propre déclassement, comme d'autres travaux le montrent (Faure, 2006). Ils opèrent une distinction entre eux et les autres, ces "nouveaux», «toutes ces familles qu'on a mis ces dernières années », certains désignant plus directement les «immigrés», les « Noirs 》. Ces classements et catégorisations les repositionnent en haut ou au milieu de la hiérarchie sociale locale.

La perspective au moment de la démolition est différente selon que ces ménages sont en couple, ou sont des personnes seules. Les premiers ont encore des projets de départ, de retour au pays, ou d'allers-retours entre le pays d'origine et le pays d'accueil, quand les deux seront à la retraite, quand le dernier enfant aura terminé ses études... Les seconds veulent plutôt rester, ils ont leurs habitudes et sont plus isolés. Ayant vécu dans des logements vétustes et d'urgence et confrontés dans certains sites à des démolitions successives engagées depuis la fin des années 1980, plusieurs de ces familles ont déjà été relogées au moins une fois. Ces trajectoires de «relogés » représentent $8 \%$ de l'ensemble des ménages que nous avons interrogés. Par ailleurs, la démolition intervient en fin de 
trajectoire résidentielle, incitant certains de ces ménages à mettre en œuvre plus rapidement un projet de retour dans sa région d'origine pour éviter de vivre un relogement incertain et traumatisant.

\section{Encadré 5 : Trajectoire résidentielle type « vieille famille ouvrière » \\ - 1950/1960: \\ Hébergement familial, habitat précaire (caravane, hôtels) \\ - 1960/1970:}

Premier logement HLM dans la ZUS ou dans une autre ZUS proche (cité d'urgence, logement sousnormé) - correspond à la naissance des premiers enfants. L'accès au logement social permet de sortir de l'habitat précaire et temporaire et d'accéder à plus de confort.

$-1959 / 1975$ :

Logement dans la ZUS (avec une moyenne de 2,6 logements occupés avant le relogement), relogement suite à des démolitions de cités d'urgence ou d'immeubles insalubres

- 1989/1999:

Mutations internes au fur et à mesure des naissances (pour tous), trajectoire dans la ZUS

- 1996/2001 :

Premiers relogements ZUS 2

- 2002 / 2006 :

Relogements plus récents, relocalisation hors ZUS ou dans des "petits bâtiments "

Retour province / résidence définitive (personnes seules en particulier) : certains ménages préfèrent partir d'eux-mêmes et accélérer un projet résidentiel qu'ils avaient en tête

Ancrage local de la famille (parents, enfants)

Encadré 6 : Trajectoire résidentielle type « vieille famille ouvrière à parcours migratoire »

- 1960/1970 :

Arrivée de l'homme seul, migration travail

- 1970/1980 :

Regroupement ou/et installation famille, accès à un logt autonome : privé (6), social (2)

1978/1992 : accès à un logement social ZUS et ajustements taille

1989/1999 : mutations internes au fur et à mesure des naissances (pour tous), trajectoire dans la ZUS

- 1996/2001 :

Premiers relogements ZUS

- 2002/2006 :

Relogements plus récents, relocalisation hors ZUS ou dans des «petits bâtiments »

Retour au pays/ résidence définitive (personnes seules en particulier)

\section{Les trajectoires de ménages "fragilisés " et de "grandes familles " sédentaires}

Le deuxième type, celui que nous avons appelé les trajectoires de ménages fragilisés et de " grandes familles" (type 2, 38\% des ménages interrogés) rassemble des ménages qui ont en commun une faible propension à la mobilité. Cette dernière est liée au cumul de faibles ressources, ou/et au fait d'avoir beaucoup d'enfants, et pour certains, à une origine étrangère qui les confronte à la discrimination et ne facilite pas leur accès à d'autres types de quartiers. Tous ont un profil de sédentaires et pour tous, le quartier a été et reste une ressource de logement. Deux catégories de ménages assez différents relèvent de ce type de trajectoires : des familles immigrées avec plus de trois enfants, dont les pays d'origine sont variés et des familles monoparentales pauvres. C'est parmi les ménages de ces trajectoires qu'on trouve le niveau de revenus le plus faible, les situations de suroccupation du logement et des situations de fort endettement. Une grande part de leurs ressources provient d'aides et d'allocations.

Ces trajectoires résidentielles sont plus diversifiées et chaotiques, avec davantage d'étapes (logement privé, hébergement...). L'arrivée dans le quartier est plus récente que celle des «vieilles familles ouvrières ». Elle s'échelonne des années 1980 aux années 1990, avec pour les familles immigrées, une arrivée entre 1985 et 1995. L'accès au quartier peut avoir été vécu comme une promotion (grandes familles mal logées) ou une régression (ruptures familiales). Mais quelles que soient la filière et les circonstances d'arrivée, le logement social a permis à tous d'atténuer leurs difficultés, de solutionner un problème de logement ou de précarité familiale. Les trajectoires des familles monoparentales et de personnes seules fragilisées sont avant tout le reflet de ruptures et d'un 
cumul de difficultés familiales, psychologiques et financières qui marquaient déjà pour certains leur enfance et leur adolescence. C'est parmi ces familles monoparentales et personnes fragilisées que les séquences résidentielles sont les plus nombreuses. Les passages par des foyers, des résidences, de la location privée et plusieurs logements sociaux sont fréquents. Étant donné leur situation et le faible loyer que ces ménages paient, rester dans le logement social est une question de survie. Les couples d'immigrés ayant plus de trois enfants ont connu l'hébergement dans le quartier ou dans le parc privé insalubre, chez des proches parents, avant d'obtenir un logement social autonome. Les trajectoires résidentielles et sociales ne sont pas forcément marquées par les mêmes ruptures mais plutôt par une absence de choix. L'arrivée dans le quartier s'est faite par réseau familial et a été un moyen de loger sa famille. Ces ménages ont un fort ancrage communautaire local, à l'échelle du quartier plus que de l'immeuble, voire souvent de la commune.

Le rapport à l'espace local des ménages qui ont ces trajectoires est ambivalent. Dans les classements sociaux et symboliques de la cohabitation locale, ces groupes sont stigmatisés. Rendus responsables de "la dégradation du quartier», ils ont peu de marges de manœuvre pour gérer des distances sociales. L'inscription dans des réseaux sociaux fait la différence. Ceux qui ont un espace large de sociabilité de liens forts de type «communautaire » ont un ancrage plus positif. Les ménages qui cumulent précarité et isolement social ont un rapport plus négatif avec l'espace local, fait d'isolement et de peur. L'expression « je me sens dépassé (e) » est souvent utilisée pour décrire des situations de cumul de difficultés. Le relogement s'inscrit dans des histoires personnelles chaotiques, est plus subi que choisi et intervient au milieu de la trajectoire résidentielle.

Encadré 7: Trajectoire résidentielle et familiale type "grandes familles sédentaires" et « ménages fragilisés » - la ZUS comme lieu d'arrivée et d'ancrage

$-1980 / 1990$ :

Arrivée en France de l'homme seul (ou de l'ensemble de la famille pour quelques uns). Hébergement dans la ZUS (principale filière d'accès). Trouve du travail

- 1985/1995 :

Arrivée de la famille - Poursuite de l'hébergement (l'un d'entre eux a été hébergé pendant trois ans) ou accès à un logement autonome dans la ZUS

- Années 1990 :

Naissance d'autres enfants et éventuelle mobilité vers un logement plus grand ou vers un logement social

- Années 2000:

Relogement : mutation, décohabitation, même type de logement

Ancrage local communautaire fort à l'arrivée et par la suite: association musulmane indienne, communauté comorienne...

\section{Les trajectoires ouvertes de "petits ménages salariés"}

Le dernier type correspond à des trajectoires plus ouvertes de petits ménages actifs salariés (Type 3, 36,4\% des ménages interrogés). Ces ménages se caractérisent par leur jeunesse, par le fait qu'ils en sont plutôt au début de leur trajectoire et par une situation économique plus favorable. Ce sont des personnes seules et des couples avec moins de trois enfants ou sans enfant, dont le chef de ménage a moins de 40 ans et dont au moins un des membres a un emploi. Tous ont en commun une taille de ménage assez réduite et des revenus d'emplois plus que d'assistance. On peut distinguer trois sous-groupes selon les circonstances d'arrivée dans le quartier et les liens qu'ils y ont noué. Le premier est celui que nous avons appelé les "enfants de la cité » en reprenant l'auto-désignation d'un des ménages interrogés. Ils ont en commun d'être nés là ou d'y être arrivés très jeunes avec leurs parents. C'est une deuxième génération d'habitants et d'enfants d'immigrés, un peu plus diplômés et qualifiés que leurs parents. Le deuxième sous-groupe est celui des "immigrés récents 》, qui sont depuis peu de temps en France (arrivée dans le «quartier» à partir du milieu des années 1990) et dont la situation typique antérieure ou en cours est celle de l'hébergement par des proches dans le quartier. Venant de pays d'immigration variés pour des raisons politiques et économiques (Sri-Lanka, Inde, Turquie...), ils ont là encore des perspectives incertaines de jeunes ménages au début de leur trajectoire, mais sont salariés. Enfin, le troisième sous-groupe correspond à des salariés occupant des emplois peu qualifiés dans la restauration et le nettoyage, qui ont peu d'attaches au quartier. Étant 
donné leurs faibles revenus, le logement social leur permet de mieux vivre. Les étapes antérieures de la trajectoire de l'ensemble de ces ménages sont peu nombreuses : logement dans la famille pour « les enfants de la cité », hébergement pour les jeunes immigrés arrivés récemment, premier logement par le $1 \%$ patronal pour d'autres... Le logement social a été et/ou est un tremplin avant d'autres étapes résidentielles. Le relogement intervient au début de leur parcours résidentiel et de parcours familiaux et professionnels en construction. Leurs pratiques urbaines et sociales s'inscrivent dans des espaces larges. Cette autonomie par rapport à l'ancrage local, leur plus grande projection dans un avenir ailleurs et dans des projets professionnels, les rend beaucoup plus ouverts à la mobilité, urbaine et résidentielle. Insérés pour certains dans des réseaux locaux de type communautaire, ils cherchent une «bonne distance » avec le poids des normes de ces grappes familiales. Leur pratique du quartier est plutôt du côté d'un "quartier d'individus» (Authier, 2010) et c'est en tout cas plutôt à cela qu'ils aspirent.

\section{Encadré 8 : Trajectoire résidentielle type « petits ménages actifs » et « immigrés récents »}

\section{- 1980/1990:}

Arrivée homme seul (et regroupement familial après), ou arrivée avec la famille,

Hébergement pendant plusieurs mois, voire plusieurs années, au sein de la ZUS, avec une variante qui est la location dans le parc privé (accès aux Bosquets également) et une autre qui est la co-location familiale

- 1995/1997 :

Accès à un logement social $=$ logement autonome

Mutation interne pour un logement plus grand que le relogement peut favoriser

- Années 2000 :

Relogement : accession, mutation interne

\section{Une autre approche du changement et des effets sociaux de l'action}

L'analyse de la mobilité contrainte à partir des trajectoires permet aussi et surtout de mettre en relief les sens différents que les ménages vont donner à ce changement. Les attentes, les enjeux et les opportunités ne sont pas les mêmes. Les trajectoires différencient à la fois les ressources relationnelles et matérielles, les capacités de négociation, leurs rapports avec les institutions locales et les interactions avec les agents chargés du relogement. Le sens des notions d'opportunité, d'amélioration et de dégradation des situations résidentielles se redéfinit différemment dans les trois types de trajectoires.

\section{"L'autochtonie " des vieilles familles ouvrières; un relogement négocié}

Le concept de capital d'autochtonie (Retière, 2003) est pertinent pour analyser à la fois le rapport à l'espace local des «vieilles familles ouvrières » et leur rapport au changement. Ce capital d'autochtonie s'est constitué avec leur ancienneté de résidence, un ancrage familial "dans le coin », de leurs parents mais aussi et surtout de leurs enfants (ou les deux). Ils ont à la fois les acquis d'une culture ouvrière faite de «localisme et de familialisme » (Verret, 1979) et des ressources liées à leur ancien engagement associatif, politique. Ils vont les faire jouer dans l'interaction du relogement, et cela de plusieurs façons.

Tout d'abord, leur connaissance fine de la géographie sociale des lieux leur permet de mieux choisir leur relocalisation ou d'être en mesure de refuser certaines propositions. Le temps et leur position ont favorisé la construction de relations privilégiées avec du personnel municipal et de l'organisme HLM. Ensuite, ce capital d'autochtonie est avancé dans la négociation comme un argument pour faire valoir un "mérite» du relogement. Ils jouent sur deux statuts, celui de "bon locataire » et celui «d'engagé, de médiateur». Par ailleurs, ceux qui s'étaient retrouvés au fur et à mesure isolés dans des immeubles dont le peuplement ne leur ressemblait plus, vont essayer de faire reconnaître une situation résidentielle « injuste » de déclassement, par la plainte et la revendication. Ils se positionnent face aux gestionnaires dans des rapports de réciprocité et dans une position de méritants du relogement qui leur donne droit à un traitement préférentiel.

Que veulent-ils obtenir et qu'obtiennent-ils ? Ils veulent plutôt rester, au moins pendant encore quelques années, mais profiter de ce relogement pour améliorer leur position résidentielle. Leurs stratégies, car il y a bien stratégie pour une partie d'entre eux, sont orientées autour d'une même 
préoccupation : maintenir ou/et améliorer son voisinage, ce voisinage étant un enjeu de distinction et/ou un risque de déclassement social. Le lieu de relogement prime sur la taille ou le loyer. Si opportunité il y a, elle est plutôt de « sortir de la cité » pour ceux qui se sentent au cœur des problèmes de délinquance et de drogue et vivent mal leur voisinage, ce qui veut dire le plus souvent accéder à un "secteur calme » dans des petits bâtiments, ou s'assurer de rester avec ses proches pour ceux qui se sentaient bien dans leur ancien immeuble. Cet enjeu est si fort pour eux, qu'il peut conduire à de l'action collective. Ainsi, une dizaine de ces vieilles familles ouvrières maghrébines, déjà relogées une fois pour la plupart, n'ont posé qu'une condition au bailleur d'une des opérations, pour accepter de partir : être relogées ensemble juste à côté, surtout pas dans un autre secteur. Le contrôle social exercé sur l'accès au petit immeuble rénové qui a accueilli ces familles, en dit long sur l'enjeu de la maitrise des usages de leur immeuble. Pour beaucoup, la sous-occupation des logements les conduit vers des logements plus petits, le relogement pouvant amener des désavantages. En revanche, ceux qui le peuvent contrôlent la localisation. Ils obtiennent des relocalisations dans de petits bâtiments, dans ou hors du quartier, un accès à des maisons individuelles (Corbeil), des relogements plus centraux (La Courneuve), tout en restant dans la commune. Certains parviennent à se maintenir dans un grand logement. Leur profil de petits ménages à revenus modestes, mais stables, facilite ce type de relogement (Orly). C'est parmi ces ménages que l'on trouve le plus d'amertume ou au contraire la plus grande satisfaction, selon que le relogement se traduit par la perte d'un «bon voisinage » ou par l'obtention d'un « meilleur voisinage ».

\section{La dépendance des sédentaires fragilisés : un relogement d'adaptation subi}

Pour les ménages relevant du deuxième type, la situation et le sens du relogement s'inscrivent dans des trajectoires antérieures subies et dans un rapport de dépendance très fort aux institutions locales. Ils sont aussi sédentaires, ayant très peu de capacités de mobilité et de projets résidentiels. Le relogement n'est pas investi de projections comme dans les deux autres types. L'enjeu pour tous est le même: préserver son logement et une marge de manœuvre financière pour vivre ou survivre, autrement dit éviter une augmentation de la dépense et conserver un pouvoir d'achat. Le voisinage est secondaire au moment du relogement même si, dans une temporalité plus longue, il peut devenir la cause première de leur insatisfaction.

La préoccupation de préserver un bas niveau de dépense pour le logement rencontre celle des bailleurs, l'échange se faisant sur la base de cette gestion mutuelle des risques. Les agents chargés du relogement leur rappellent souvent l'éventail limité de leurs choix, et eux-mêmes l'intègrent en n'émettant pas de souhaits de départ, et en n'attendant pas grand bénéfice de ce relogement. Même s'ils peuvent avoir des familiarités avec les lieux, ils ont beaucoup moins de marge de manœuvre pour négocier. Peu utiles pour négocier leur relogement, les liens forts d'appartenance à des réseaux sociaux élargis permettent à certaines familles de mieux vivre que d'autres le déménagement dans des bâtiments très concentrés en " grandes familles immigrées » et en populations pauvres. Par ailleurs, le fait d'être dans les lieux au moment du relogement peut transformer des hébergés en «ménages à reloger », ce statut leur donnant priorité pour l'accès à des nouveaux logements autonomes. Le fait d'être dans les lieux leur garantit le relogement malgré des situations d'endettement et de suroccupation.

S'ils ne sont pas mis et ne se sentent pas dans une position de négociation, ils peuvent néanmoins bénéficier d'opportunités, liées aux pratiques d'adaptation par les relogeurs du logement à la taille et aux capacités financières du ménage : un logement autonome pour les enfants, un logement plus grand ou encore l'apurement de leur dette. Certains ménages parviennent ainsi à faire aboutir des demandes de logement et de mutations qui jusque-là n'avaient pas été prises en compte. L'ancrage peut améliorer leur situation résidentielle et le relogement peut leur donner accès à des liens institutionnels facilitant leur accès à des formations professionnelles ou remettant à jour leurs droits sociaux. Mais le saut qualitatif d'un apurement de la dette ou de l'agrandissement du logement n'est pas du même ordre que celui d'un accès à un logement neuf ou d'une « sortie de la cité », qui concerne peu ce type de ménages, sauf lorsque les grands logements sont localisés ailleurs et qu'ils subissent cette « sortie ». Il ne change pas grand-chose au sens de ces trajectoires résidentielles et sociales subies et contraintes. Pour les familles les plus fragiles, cette situation de dépendance et la priorité donnée à la dépense de logement font accepter des propositions qui accentuent leur isolement. L'amertume de certaines familles monoparentales traduit un certain désarroi, quelque temps après le relogement, 
devant un voisinage qui est trop distant, ou dont on craint au contraire la proximité spatiale et sociale. Être dans un logement social et y rester est une nécessité qu'ils cherchent avant tout à préserver. On retrouve des comportements et types de relation propre aux "fragiles» et aux "assistés ", "deux idéaux-types » construit par S. Paugam pour analyser les rapports entre les populations pauvres et les services sociaux (Paugam, 1996).

\section{L'autonomie des petits ménages salariés, passants et mobiles : des opportunités résidentielles}

Les actifs salariés sont inscrits dans des réseaux à la fois locaux (familiaux et institutionnels) et extra-locaux (travail, loisirs). Leurs trajectoires sont plus ouvertes, la négociation est également plus aisée, le relogement souvent source de satisfaction. Ces ménages négocient leur relogement, et ce d'autant plus facilement qu'ils correspondent assez bien au profil de ménages que les bailleurs et les communes voudraient voir rester, à la catégorie idéale «du parcours résidentiel positif» de la rénovation. Ils négocient leur relogement, font des demandes et ne se contentent pas de répondre à des propositions. Ils se débrouillent aussi par eux-mêmes. Leurs aspirations sont plus hétérogènes. Pour les groupes dont l'ascension sociale est en route ou vivement souhaitée, l'enjeu va être d'accéder à un autre environnement social pour élever leurs enfants. D'autres vont profiter de cette situation pour réaliser un projet résidentiel d'achat, activer les réseaux de leur employeur et se rapprocher de leur travail ou demander un logement plus grand. Pour les enfants et proches hébergés, l'enjeu va être celui de l'accès à un logement autonome et à un logement social. Le relogement peut alors conforter une trajectoire sociale ascendante et donner un sens plutôt promotionnel à leur trajectoire résidentielle, en particulier lorsqu'ils partent ailleurs, ou lorsqu'ils accèdent à un logement neuf. Pour beaucoup, les conditions de logement plus avantageuses se conjuguent avec un changement de voisinage. La relocalisation tend plutôt à les faire accéder à « des types résidentiels » mieux placés dans la hiérarchie spatiale et sociale, que ce soit en quittant «la cité » (terme utilisé par les ménages eux-mêmes) ou en passant d'une barre à un petit bâtiment neuf au sein du même quartier. Minoritaires, ces trajectoires pourraient somme toute s'apparenter à des trajectoires ascendantes assez classiques, inscrivant le changement résidentiel dans une trajectoire sociale promotionnelle (changement d'emploi, augmentation de salaire...). La différence est néanmoins double: d'une part ce n'est pas le changement de position sociale qui détermine cette mobilité mais la mobilité contrainte qui peut favoriser des ajustements résidentiels; d'autre part, ces jeunes salariés restent des ménages à revenus modestes, voire bas pour les plus jeunes et les hébergés. Décohabitation, relogement dans le neuf, sortie de la cité, accession sont autant d'opportunités offertes par la rénovation et mobilisables par ces ménages. Le changement résidentiel est de fait un changement à la fois de conditions de logement et d'environnement. Il se conjugue pour certains d'entre eux avec une trajectoire d'autonomisation vis-àvis des parents et proches et d'ascension professionnelle.

\section{Conclusion}

Les trois types de trajectoires identifiées au sein des ménages relogés suite aux démolitions dans les quartiers rénovés permettent de montrer le sens et les effets différenciés de cette mobilité contrainte. Ils mettent également en exergue deux autres processus. Cette approche par les trajectoires rend compte d'une part de l'hétérogénéité des classes populaires vivant dans ces quartiers et peut constituer en ce sens un cadre d'analyse du peuplement de ces territoires et pas seulement des trajectoires des relogés. Elle éclaire d'autre part un processus de changement social très différent de celui à l'œuvre dans d'autres types de « rénovations », comme celle des années 1960 (Coing, 1966) ou dans des transformations urbaines de quartiers anciens et qualifié le plus souvent de "gentrification » (Authier, 1996 ; Bidou-Zachariasen, 1996 ; Dansereau, 1985).

Contrairement aux représentations homogénéisantes du peuplement des quartiers concernés par la rénovation urbaine, il existe encore une hétérogénéité des trajectoires résidentielles et des perspectives qui feront rester ou partir les ménages qui y vivent. Cette hétérogénéité n'a certes pas les mêmes composantes que celle des années 1970, qui faisait cohabiter dans les grands ensembles l'éventail des salariés français et se caractérisait par une "quasi absence des générations âgées " (Chamboredon, Lemaire, op. cit.). Elle rend compte d'une évolution plus générale qui a conduit à la fois au vieillissement de la population et à la «fragmentation» de la classe ouvrière (Collewald, Schwartz, 2006). 
Les trajectoires des vieilles familles ouvrières montrent à quel point cette fraction du logement social que sont les grands quartiers d'habitat social des années 1960/1970 a été et reste le lieu d'habitat de toute une génération de salariés des «trente glorieuses». Ces ménages forment en partie la « couche supérieure des classes populaires ou inférieure des classes moyennes » (Dubet, 1997). Leurs trajectoires ont une proximité avec celles «des petits moyens » qui sont allés acheter un pavillon pas loin dans les années 1980 et 1990, leurs anciens voisins qui ont fait le choix que eux-mêmes n'ont pas pu ou pas voulu faire à ce moment-là (Cartier et alii, 2008). La rénovation peut accélérer des projets résidentiels de fin de trajectoire, les amenant à partir dans leur résidence secondaire ou à rester tout en profitant des opportunités d'achat ou de relocalisation préférentielle.

Les trajectoires des ménages plus fragilisés correspondent assez bien à celles des «anciens membres des classes populaires autrefois intégrés sur le marché du travail et qui, fragilisés par tel ou tel accident biographique (maladie, séparation, divorce...), en ont été expulsés, voyant ainsi leur vie basculer dans une grande vulnérabilité sociale» (Beaud, Pialoux, 2003, p. 97). Elles confirment la spécialisation de cette fraction du parc social dans l'accueil de ménages "pauvres 》 et «déstabilisés 》 mais aussi de familles immigrées, pour qui elle a été et reste le seul moyen d'accès au logement social (Simon, 1998). Les trajectoires de ménages fragilisés et de "grandes familles » témoignent d'une immigration plus récente et plus pauvre ainsi que des évolutions plus sociétales de la famille. Ces transformations de la famille ont aussi conduit des femmes peu qualifiées, parfois sans autre ressource que l'aide de la famille ou les aides sociales, dans ces logements sociaux. Ces ménages correspondent au groupe des «ménages totalement dépendants des aides»(Dubet, op. cit.). Pour ceux-là, la rénovation au mieux ajuste des situations de sur-occupation, au pire dégrade leur situation et surtout ne change pas grand-chose.

Quant aux «petits ménages actifs », leur présence dans ces bâtiments démolis confirme la permanence de trajectoires de passage par ces espaces et le caractère impropre du terme de ghetto, du moins pour décrire une réalité de peuplement plus complexe et plus variée (Wacquant, 2005). Ces trajectoires reflètent également le maintien d'une fonction d'accueil du salariat qui a été la vocation d'origine du logement social. Dans ce type de trajectoires, se mêlent deux strates de la population de ces quartiers, des "familles qui disposent d'un revenu relativement élevé et d'un emploi salarié stable », qui correspondent à la "population traditionnelle du logement social» et «les salariés les plus mal payés qui ne peuvent envisager de quitter la cité », des « salariés pauvres et précaires » dont les emplois sont « incertains» (Dubet, 1997). Ces derniers ont plus de mal à quitter le logement social dans la mesure où le loyer faible permet de vivre et de faire face aux aléas de leur emploi. Mais leur aspiration n'est pas forcément de finir leur vie là et les changements familiaux ou l'amélioration de leur situation peuvent les amener à bouger.

Ces trois types de trajectoires ont toutefois un point commun. Pour tous ces ménages, l'arrivée dans le logement social et dans le quartier, quelle que soit la période, s'est traduite par une amélioration de leur situation résidentielle et constitue une "garantie sociale » face aux risques de « désaffiliation » (Castel, op. cit.).Ces trajectoires éclairent par ailleurs des processus de changements sociaux liés à la démolition-reconstruction très différents de ceux des années 1960 et non sans intérêt au regard cette fois des objectifs de l'action publique. Une des critiques sociologiques fortes faites à ces opérations est de viser à travers la mixité sociale la dispersion des immigrés (Kirszbaum, 2002) amenant soit à des effets inverses, soit/et à une sorte de «double peine», celle d'un déplacement contraint et socialement déstructurant et celle d'une gentrification d'un quartier populaire réinvesti par les couches moyennes et supérieures. Sans nier la diversité des stratégies et situations locales, l'approche par les trajectoires déconstruit tout d'abord la catégorie «immigrée ». Elle tend à montrer surtout comment les vieilles familles ouvrières immigrées originaires des pays du Maghreb ou du Sénégal mais également du Chili, du Portugal ou d'Asie d'une part et les enfants d'immigrés d'autre part peuvent bénéficier et se saisir des opportunités résidentielles pour sortir du quartier ou au contraire s'y ancrer. Les travaux plus récents que nous avons menés sur les nouveaux arrivants dans les programmes neufs de logements privés dans trois quartiers confortent encore cette perspective de changement endogène par des trajectoires promotionnelles internes stabilisant les « élites immigrées », petites classes moyennes locales. Ainsi, on retrouve dans la clientèle de la promotion privée, et en particulier dans l'accession sociale, quelques trajectoires de vieilles familles ouvrières mais surtout des 
trajectoires «d'enfants de la cité » ${ }^{3}$ (Lelévrier, 2011 ; Saint-Macary, 2011). Les opportunités de la rénovation n'en restent pas moins inégales et inégalement mobilisables.

\section{Bibliographie}

ALLEN, T., 2000, “Housing renewal- doesn't it make you sick ? ", Housing Studies, 15, p. 443-461.

AUTHIER J-Y. (dir.), 2010, État des lieux sur les trajectoires résidentielles, PUCA.

AUTHIER J-Y., 1996, « Mobilités résidentielles et effets de composition dans les processus de réhabilitation des quartiers anciens centraux », in HAUMONT N., (dir.), La ville: agrégation et ségrégation sociales, L'Harmattan.

BEAUD S., PIALOUX M., 2003, Violences urbaines, violence sociale, genèse des nouvelles classes dangereuses, Fayard.

BIDOU-ZACHARIASEN C., 1996, « Classes populaires et classes moyennes en centre historique rénové », in N. HAUMONT (dir.), La ville : agrégation et ségrégation sociales", L’Harmattan, p. 131-142.

BOLT, G., PHILIPS, D. \& VAN KEMPEN, R., 2010, "Housing policy, (de) segregation and social mixing : an international perspective", Housing Studies, Vol. 25, n² 2, p. 129-135.

BONVALET C., FRIBOURG A-M. (eds.), 1990, Stratégies résidentielles, Paris, Plan Construction, INED.

CARTIER, M. COUTANT, I. MASCLET, O., \& SIBLOT, Y., 2008, La France des "petits moyens", enquête sur la banlieue pavillonnaire, Paris, La Découverte-

CASTEL R., 1995, Les métamorphoses de la question sociale, une chronique du salariat, Paris, Fayard.

CHAMBOREDON, P. \& LEMAIRE, M., 1970, "Proximité spatiale et distance sociale. Les grands ensembles et leur peuplement", Revue française de sociologie, Vol. XI, ${ }^{\circ} 1$.

CHARBONNEAU J., 1998, «Trajectoires sociales et stratégies individuelles en contexte d'incertitude », in Y. Grafmeyer, F. Dansereau, Trajectoires familiales et espaces de vie en milieu urbain, PUL.

COLLEWALD A., SCHWARTZ O., 2006, Entretien avec, "Haut, bas, fragile, sociologies du populaire », Vacarme, $\mathrm{n}^{\circ} 37$.

COING, H. , 1966, Rénovation urbaine et changement social, Paris, les Éditions Ouvrières.

DANSEREAU F., 1985, «La réanimation urbaine et la reconquête des quartiers anciens par les couches moyennes : tour d'horizon de la littérature nord-américaine », Sociologie du travail, n 2, p. 191-205.

DONZELOT, J. (dir.), 2012, À quoi sert la rénovation urbaine?, PUF.

DUBET F., 1997, «Comment nommer les “classes populaires” », collectif, Ces quartiers dont on parle, L'Aube, p. 39-65.

FAURE S., 2006, "De quelques effets sociaux de la démolition d'immeubles, un grand ensemble HLM à SaintÉtienne, Espaces et sociétés, $\mathrm{n}^{\circ}$ 124-125, p. 191-205.

FOL S., 2009,La mobilité des pauvres, Paris, Belin.

GOETZ, E.G., 2002, "Forced relocation v.s voluntary mobility, the effects of dispersal programm on households", Housing studies, $\mathrm{n}^{\circ}$ 17, p. 107-123.

GOUX, D. MAURIN, E., 2007, "Close Neighbours matter: Neighbourhood effects on early performance at school”, Economic Journal, n 117, p. 193-215.

GRAFMEYER, Y., 1994, Sociologie urbaine, 128, Nathan, Paris.

HUYNH, P.M., 2008, Démolitions-reconstructions et trajectoires résidentielles des ménages, État des savoirs et perspectives d'action, coll. recherche, PUCA.

KLEINHANS R., VAN DER LAAN BOUMA-DOFF W., 2008, “On priority and Progress, : forced residential relocation and housing chances in Haaglanden, the Netherlands", Housing studies, Vol. 23, n 4, p. 565-587.

KLEINHANS, R., 2004, "Social implications of housing diversification in urban renewal : a review of recent literature", Journal of Housing and the Built Environment, p. 367-390.

KLEINHANS, R., 2003, "Displaced but still moving upwards in the housing career ? implications of forced residential relocation in the Netherlands", Housing Studies, Vol. 18, n 4, p. 473-499.

LELEVRIER, C. NOYE, C., 2012, "La fin des grands ensembles », in DONZELOT, J. (dir.) A quoi sert la renovation urbaine, PUF, Paris, p. 185-221.

LELEVRIER, C. (dir.), 2011, Diversification de l'habitat et mixité sociale, rapport PUCA.

LELEVRIER C., 2010, "La mixité dans la rénovation urbaine : dispersion ou re-concentration ?" Espaces et Sociétés, Vol. 140-141, n 1-2, p. 59-74.

LELEVRIER, C., 2008, Mobilités et trajectoires résidentielles des ménages relogés lors d'opérations de renouvellement urbain, synthèse de travaux menés entre 2004 et 2007, PUCA/DIV/DREIF.

LELEVRIER, C (dir.), 2007, Mobilités et trajectoires résidentielles des ménages dans trois opérations de rénovation urbaine en Ile-de-France, PUCA/C.R.E.T.E.I.L.

LELEVRIER, C. NOYE, C., 2007, L'observation des mobilités résidentielles liées aux démolitions dans le cadre de la rénovation en Ile-de-France, rapport DREIF.

\footnotetext{
${ }^{3}$ L'article d'Emilie Saint-Macary dans le présent ouvrage met en évidence ce type de trajectoires.
} 
LEVY J-P., 1998, "Habitat et habitants : position et mobilité dans l'espace résidentiel”, in Grafmeyer Y., Dansereau F., Trajectoires familiales et espaces de vie en milieu urbain, PUL.

OBLET T., VILLECHAISE A., 2009, "Les relogés de la rénovation : un bilan mitigé", Mouvements, revue en ligne.

PERALDI M. FORET C., 1992, Le sens des trajectoires, CNAF.

RETIERE J-N., 2003, “Autour de l'autochtonie. Réflexions sur la notion de capital social populaire", Politix, Vol. 16, n 63, p. 121-143.

SARI, F., 2012, "Analysis of Neighbourhood Effects and Work Behaviour: evidence from Paris", Housing Studies, Vol. 27, $\mathrm{n}^{\circ} 1, \mathrm{p} \ldots$

SAINT-MACARY, E., 2011, Mixité sociale et diversité de l'habitat : l'investissement d'acteurs privés dans les operations de renovation urbaine, Thèse de Doctorat sous la direction de F. Drosso \& C. Lelévrier, IUP/UPEC.

TUNSTALL, R. \& FENTON, A., 2006, In the mix: a review of mixed income, mixed tenure and mixed communities : what do we know?, report for the Joseph Rowntree Foundation, 58 p.

VARADY, D.P., WALKER, C.C., 2000, "Vouchering out distressed subsidized developments : does moving lead to improvements in housing and neighbourhood conditions ?"Housing Policy Debate, Vol. 11, $\mathrm{n}^{\circ} 1$, p. 115-162.

VENKATESH S.A.\& CELIMLI I., 2004, “Tearing down the community”, Shelterforce Online, n 318.

VERRET M., 1979,L'espace ouvrier, Paris, Armand Colin.

VIEILLARD-BARON H., 1998, "Les attributions de logements sociaux: des contraintes techniques au bricolage local », in La ville éclatée, quartiers et peuplement, sous la dir. de N. HAUMONT et J-P. LEVY, Paris, l'Harmattan, p. 175-192.

VIGNAL C., 2005, «Logiques professionnelles et logiques familiales: une articulation contrainte par la délocalisation de l'emploi », Sociologie du travail, Vol. 47, p. 153-169.

WACQUANT L., 2005, "Les deux visages du ghetto, construire un concept sociologique », in Actes de la recherche en sciences sociales, $\mathrm{n}^{\circ} 160$, p. 4-21. 